\title{
Dynamic Rigidity Percolation in Inverted Micelles
}

\author{
L. Ye, D. A. Weitz, Ping Sheng, S. Bhattacharya, J. S. Huang, and M. J. Higgins \\ Exxon Research and Engineering Co., Route 22 East, Annandale, New Jersey 08801 \\ (Received 13 March 1989)
}

\begin{abstract}
Inverted micelles are found to exhibit marked viscoelasticity with a pronounced dependence on volume fraction. The frequency dependence, measured using both ultrasonic and Brillouin techniques, reflects the dynamics of the interactions between the micelles. At low frequencies they behave as isolated spheres, while at high frequencies they behave as an instantaneous connected network. The rigidity of this network exhibits power-law scaling with volume fraction, consistent with rigidity percolation.
\end{abstract}

PACS numbers: $43.35 .+\mathrm{d}, 62.30 .+\mathrm{d}, 62.80 .+\mathrm{f}$

The elastic behavior of inhomogeneous materials provides an informative probe of their physical properties and structure. For materials comprised of random dispersions of small particles, the elastic properties are critically dependent on the connectivity, providing a sensitive measure of the nature of the bonds and the spatial correlations between the constituent particles. Similarly, the viscoelastic properties of a complex fluid can also provide new insights into the spatial correlations and interactions of its components. In this case, however, the dynamics of the fluid add new richness to the behavior.

In this Letter, we study the unusual viscoelastic properties of inverted micelles, or very small spherical aggregates of surfactant suspended in an oil. ${ }^{1-8}$ The surfactant head groups form a close packed, central core, with the tail groups oriented outwards. The volume fraction of the micelles, $\phi$, can be varied, while their radius, $a$, remains fixed. There is a weak, short-range attractive interaction ${ }^{5,6}$ between the micelles, and the characteristic time scale of this interaction, $\tau_{c}$, determines their behavior. On time scales long compared to $\tau_{c}$, their diffusive motion ensures that the micelles are dynamically dispersed in the surrounding oil. By contrast, on time scales short compared to $\tau_{c}$, the micelles form instantaneous, random clusters, which, at sufficiently high $\phi$, form a connected network. These time-dependent correlations result in a surprising viscoelastic behavior in this relatively simple fluid. We account for the $\phi$ dependence of the viscoelastic properties by means of a static effective-medium approximation for an elastic composite. We account for the frequency dependence of the viscoelastic properties through the parameters describing the micelle phase. The variation of these parameters also reflects the frequency dependence of the correlations between the micelles. At high frequencies the micelle phase exhibits a solidlike behavior which supports shear at $\phi \gtrsim 0.2$. This high-frequency rigidity of the surfactant micelle phase exhibits power-law scaling with $\phi$. We interpret this as dynamic rigidity percolation.

The inverted micelles investigated consist of spherical aggregates of AOT (sodium di-2-ethylhexylsulfosuccinate) suspended in decane. By varying the contrast of the oil, small-angle neutron scattering ${ }^{7,8}$ was used to determine that $a \approx 15.1 \AA$, corresponding to aggregates comprised of 22 AOT molecules. This size remains constant as $\phi$ is varied. ${ }^{1,3,5}$ We study the elastic properties by measuring both the speed and the damping of the longitudinal sound wave in the fluid. To establish the frequency dependence, we use both ultrasonic and Brillouin scattering measurements. For the lower frequencies, time-of-flight ultrasonic measurements were performed between 2 and $45 \mathrm{MHz}$, using an interferometric technique to measure the velocity and the decay of the echo train to measure the damping. For the higher frequencies, Brillouin scattering was performed at several scattering angles, using a five-pass Fabry-Perot interferometer with both $6471-\AA \mathrm{Kr}^{+}$and $5145-\AA \mathrm{Ar}^{+}$laser excitation. The speed of sound was determined from the peak positions of the Brillouin doublet; the damping was determined from the full width at half maximum.

The $\phi$ dependence of the speed of sound in the solution, $v$, is shown in Fig. 1 for several different frequen-

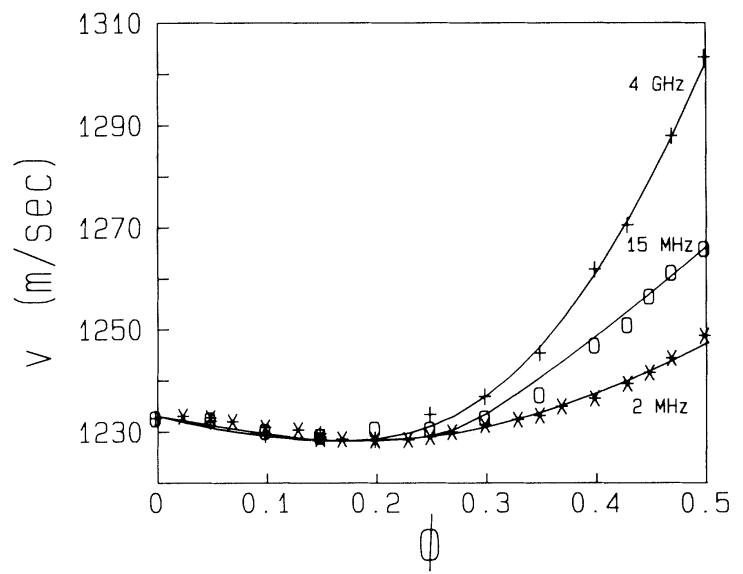

FIG. 1. Sound velocity as a function of volume fraction for several different frequencies: (*) $2 \mathrm{MHz},(0) 15 \mathrm{MHz}$, and $(+) 5 \mathrm{GHz}$. The solid lines through the data are fits by the effective-medium theory, using isolated spheres for the surfactant phase for the lowest frequency and a random, connected network for the higher frequencies. 
cies. For $\phi \leq 0.2$, there is only a slight dependence on volume fraction and no discernible frequency dependence. By contrast, for $\phi>0.2$, the speed of sound increases. Furthermore, there is a pronounced frequency dependence, with a significantly larger increase in $v$ at higher frequencies. To further investigate this dependence, the variation of $v$ on frequency, $f$, is shown in the lower part of Fig. 2 for fixed volume fraction for several values of $\phi$. The magnitude of the dispersion increases with increasing volume fraction. However, for all values of $\phi$, the dispersion occurs in the same frequency range, around $10^{8} \mathrm{~Hz}$. Furthermore, the data have reached their asymptotic limits at both the lowest and highest frequencies measured.

The initial decrease in $v$ reflects the increased density of the mixture as the volume fraction of surfactant is increased. By contrast, the increase in $v$ for $\phi \geq 0.2$ indicates an increase in the rigidity of the oil-surfactant mixture. Alternatively, the adiabatic compressibility of the mixture is decreasing, implying that the surfactant is less compressible than the oil. The remarkable feature is the pronounced frequency dependence of the increase in $v$. This implies that rigidity of the surfactant micelle phase increases at higher frequencies.

To account for the observed frequency dependence, we must consider the contribution of the surfactant micelle phase to the behavior of the mixture. We propose that it depends on the time scale of the measurement compared to the time scale of the dynamics of the micelles. This time scale arises from the short-range attractive interaction between the micelles. ${ }^{6}$ Physically, this attraction may be due to the entanglement of the tails of the neighboring spheres, ${ }^{5}$ which can interpenetrate by about $3 \AA$. At sufficiently high frequencies, the time scale of the measurement will be faster than the dynamics of this in-

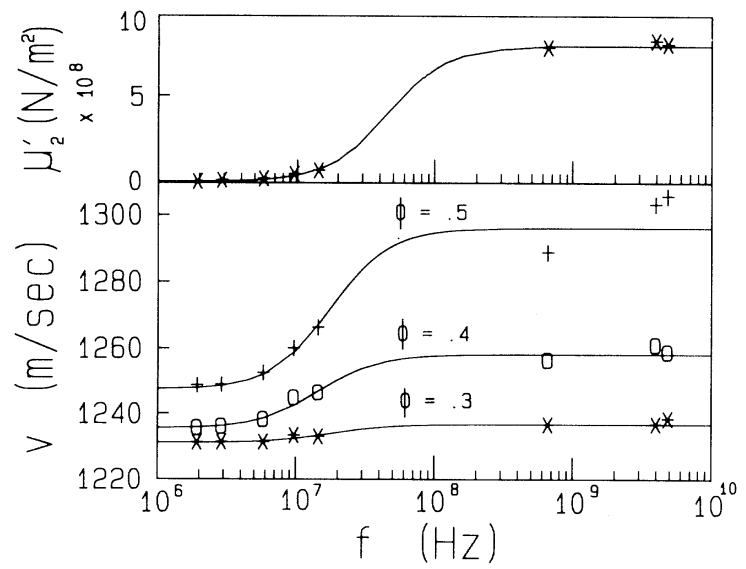

FIG. 2. Frequency dispersion of the sound velocity for several volume fractions and the real part of the shear modulus for the surfactant phase, $\mu_{2}^{\prime}$, obtained from the effectivemedium approximation. The solid lines represent the form of a single-relaxation-time Debye model. teraction and the micelle phase can be viewed as an instantaneous, connected network. This network will support shear, increasing the total fluid rigidity and hence the speed of sound. By contrast, at low frequencies, the dynamics of the micelles lead to a relaxation of any shear stress, so that the network is dynamic and the micelles behave as isolated spheres. Thus at low frequencies, there is no increase in the rigidity due to a shear modulus of the micelles.

To quantify this hypothesis, we characterize the surfactant-oil mixture by its average complex elastic constant, $\bar{\beta}^{*}=\bar{\beta}^{\prime}+i \bar{\beta}^{\prime \prime}$, which is the inverse of the adiabatic compressibility associated with the longitudinal compressional wave. The use of $\bar{\beta}^{*}$ has the advantage that it is directly related to experimentally measurable quantities. The speed of sound is obtained from the real part, $v=\left(\bar{\beta}^{\prime} / \bar{\rho}\right)^{1 / 2}$, where the $\bar{\rho}$ is the average density. Similarly, the damping of the sound wave is determined from the imaginary part, with the attenuation length for an ultrasonic propagation given by $\alpha=f \bar{\beta}^{\prime \prime} / 4 \pi v^{3} \bar{\rho}$, and the width of the Brillouin peaks given by $\Delta v=f \bar{\beta}^{\prime \prime} / 4 v^{2} \bar{\rho}$. Since $\bar{\beta}^{*}=\bar{\kappa}^{*}+4 \bar{\mu}^{*} / 3$, where $\bar{\kappa}$ and $\bar{\mu}$ are the bulk and shear moduli, the data are also sensitive to the shear rigidity of the mixture, although only the longitudinal sound propagation is measured.

The average elastic constant of the mixture contains a volume-weighted mix of the elastic constants of each of the constituent phases. Thus, we attribute both an elastic constant, $\beta_{i}^{*}$, and a shear modulus, $\mu_{i}^{*}$, to each phase, the oil $(i=1)$ and the surfactant micelles $(i=2)$. To describe the $\phi$-dependent properties of the mixture at each frequency, we use a static-effective-medium approach. This should be appropriate since the sound wavelength is always much larger than the micelle size. The dynamics of the micelles, and the resultant frequency dependence of the behavior, must be accounted for by the frequency dependence of $\beta_{2}^{*}$ and $\mu_{2}^{*}$, describing the properties of the micelle phase.

Both $\beta_{1}^{*}$ and $\mu_{1}^{*}$ can be directly determined experimentally using the pure-oil phase. Measurement of the sound velocity and damping determines $\beta_{1}^{\prime}$ and $\beta_{1}^{\prime \prime}$. Furthermore, since a fluid cannot support a shear wave, $\mu_{1}^{\prime}=0$, while $\mu_{1}^{\prime \prime}=2 \pi f \eta$, where $\eta$ is the shear viscosity at $f=0$, which is measured independently. We cannot, however, independently measure any of the constants for the second phase, as we cannot obtain a sample comprised of pure AOT micelles. Thus, there are four unknown, frequency-dependent parameters, the complex $\beta_{2}^{*}$ and $\mu_{2}^{*}$, describing the AOT micelles. These are obtained for each frequency from a fit of the data for both the sound velocity and damping to the $\phi$ dependence of $\bar{\beta}^{*}$ predicted by the effective-medium model.

At low frequencies, the dynamics of the micelles ensure that they may be regarded as noninteracting, isolated spheres, even at higher $\phi$. Thus, we use an effectivemedium model that assumes a nonsymmetric description of the mixture, ${ }^{9}$ treating the micelles as isolated spheres 
in the continuous oil phase. This results in the Wood's formula, $\bar{\beta}^{*-1}=\phi /\left[\beta_{2}^{*}-(1-\phi) / \beta_{1}^{*}\right]$, which is accurate in the static limit. ${ }^{10}$ The solid line through the data measured at $2 \mathrm{MHz}$ reflects the good fit obtained.

We are unable to fit the higher-frequency data using this model. Instead, we must assume that the micelles phase is a connected, random network which behaves as a solid, supporting shear. We therefore use a symmetric description ${ }^{9,11}$ of the structure for the two components, assuming that both the oil and the surfactant micelles form bicontinuous, intermixture phases. The symmetric description requires the role of the inclusion phase to be reversed as $\phi$ increases, and thus results in a percolation threshold. However, both components are assumed to be space filling. Since spheres cannot fill space, we have multiplied the effective-medium volume fraction by 0.64 , corresponding to the volume fraction for random close packing of spheres. ${ }^{12}$ To obtain agreement with the data requires the use of a nonzero value for $\mu_{2}^{*}$ in the fit, in accord with our physical picture. The solid lines through the data at $15 \mathrm{MHz}$ and $4 \mathrm{GHz}$ in Fig. 1 are examples of the excellent fits obtained.

The viscoelastic behavior of the mixture is reflected by the frequency dependence of $\beta_{2}^{*}$ and $\mu_{2}^{*}$. Indeed, the symmetric model used in fitting the high-frequency data reduces exactly to the Wood's formula when $\mu_{2}^{*}=0$. The frequency dependence of the value of $\mu_{2}^{\prime}$ obtained from the fits to the effective-medium theories is shown in the upper part of Fig. 2. It has roughly the same form of dispersion as the velocities, with a limiting value of $8 \times 10^{8} \mathrm{~N} / \mathrm{m}^{2}$ at the Brillouin frequencies. In addition, the bulk modulus of the micelle phase, $\kappa_{2}^{\prime}$, also exhibits a frequency dispersion, but it decreases as $f$ increases, from $1.6 \times 10^{9} \mathrm{~N} / \mathrm{m}^{2}$ at $2 \mathrm{MHz}$ to $8 \times 10^{8} \mathrm{~N} / \mathrm{m}^{2}$ at 5 $\mathrm{GHz}$. Physically, this may reflect the different properties of the micelle phase due to the time dependence of their correlations. Thus, at low frequencies, $\kappa_{2}^{\prime}$ reflects a change in volume of the isolated micelles themselves. By contrast, at high frequencies, $\kappa_{2}^{\prime}$ reflects a change in volume of the random network of micelles, which can be accomplished by changing their relative positions. This requires a smaller force than changing the volume of the micelles themselves, resulting in a reduction of $\kappa_{2}^{\prime}$. This behavior is also reflected in a decrease in $\bar{\kappa}^{\prime}$ at high frequencies for larger $\phi$. While this is highly unusual, this does not imply a violation of Le Chatelier's principle ${ }^{13}$ because $v$ still increases with frequency due to the contribution of the shear modulus, $\mu_{2}^{\prime}$. The small values of $\mu_{2}^{\prime} \approx \kappa_{2}^{\prime} \approx 10^{9} \mathrm{~N} / \mathrm{m}^{2}$ imply that the micelle network is relatively soft but nonetheless solidlike.

For $\phi \leq 0.4$, the behavior of $v$ is reasonably well described by a single-frequency Debye relaxation model, $v=v_{0}+\Delta v\left(\omega \tau_{c}\right)^{2} /\left[1+\left(\omega \tau_{c}\right)^{2}\right]$, where $v_{0}$ is the lowfrequency velocity, $\Delta v$ is the total velocity dispersion, and $\tau_{c}$ is the characteristic relaxation time. The behavior of $\mu_{2}^{\prime}$ also follows a similar form. This behavior is shown by the solid lines through the data in Fig. 2. At higher volume fractions, the velocity dispersion is no longer well described using only one relaxation time. Nevertheless, the characteristic range of relaxation times for all the data is $\tau_{c} \approx 10^{-8} \mathrm{sec}$. Within our picture, $\tau_{c}$ reflects the dynamics of the micelles, and corresponds to their characteristic interaction time. We can test this hypothesis by using oils of different carbon chain lengths, which changes ${ }^{6}$ the interaction energy, $E_{a}$, and thus $\tau_{c}$. Replacing the decane with hexane decreases $E_{a}$, and we observe no dispersion in $v$, even at $\phi=0.5$. This corresponds to a decrease in $\tau_{c}$ below our accessible range. By contrast, replacing decane with hexadecane increases $E_{a}$. In this case, we observe an immediate rise in $v$ at the lowest accessible frequencies for $\phi=0.5$, corresponding to an increase in $\tau_{c}$. We note that the changes in $\tau_{c}$ are much greater than the changes in the viscosity of the oils, implying that the dynamics reflect the interaction time, rather than merely a Brownian diffusion time.

The dynamics of the inverted micelles lead to the unique behavior of this complex fluid. At low frequencies their dynamics ensure that the micelles behave as independent, isolated spheres even at high $\phi$. By contrast, at higher frequencies, this is an ideal system to use to measure the elastic properties of random inhomogeneous mixtures. The fact that they are a fluid ensures a truly random dispersion of spheres. The fact that we can measure the speed of sound at both low and high frequencies ensures that we can distinguish the additional contribution of the rigidity of the micelle network. The difference between the high- and low-frequency moduli, $\Delta \bar{\beta}$, is, to lowest order, linear in the change in $\mu_{2}^{\prime}$, and thus reflects the rigidity of the micelle network. We are able to discern a change in $v$ only at volume fractions above $\phi_{c} \approx 0.16$. Thus, we plot $\Delta \bar{\beta}$ as a function of $\phi-\phi_{c}$ on a logarithmic plot in Fig. 3. The resolution of the Brillouin peaks precludes measurements closer to $\phi_{c}$. Never-

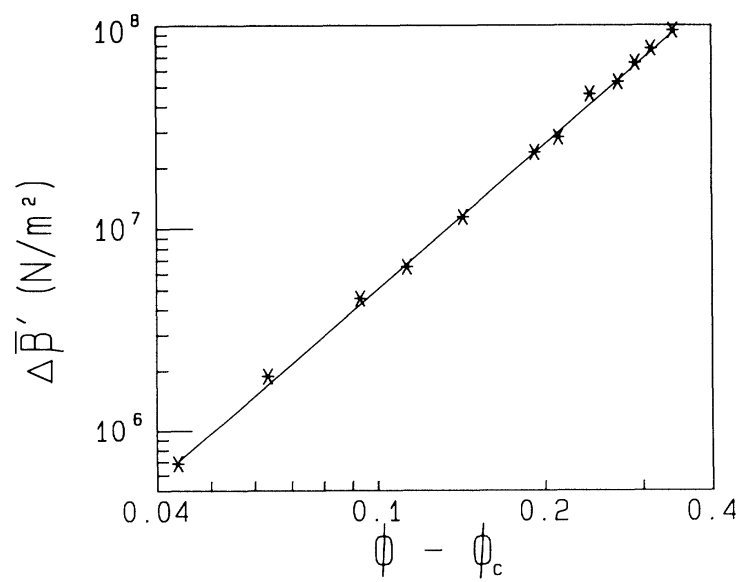

FIG. 3. Critical behavior of the shear modulus contribution of the surfactant phase at high frequencies, when it forms a random connected network. The exponent is $\tau \approx 2.5$ and $\phi_{c} \approx 0.16$. 
theless, we observe a clear power-law behavior, with a rigidity exponent of $\tau \approx 2.5$. This scaling extends over a rather large range in $\phi-\phi_{c}$, and is strongly suggestive of the behavior of a random percolating network. We emphasize that this observation is based solely on the experimental data, independent of any model. However, this is a dynamic rather than a static measurement, which limits the spatial extent of the sensitivity to the rigidity to roughly the wavelength of the sound, which is nonetheless at least $100 a$ at the highest frequency. Finally, this rigidity transition occurs only for the surfactant micelle phase, which forms the random connected network. The surrounding oil phase ensures that the mixture maintains a finite bulk modulus at all $\phi$ and does not exhibit a percolation threshold.

There are relatively few results available for elasticity percolation of random networks in three dimensions with which these data can be compared. No simulations or calculations for random, multiply connected threedimensional networks exist. However, it is known that the electrical conductivity ${ }^{14}$ scales with an exponent $t \approx 2$ in three dimensions, with $\sigma=\left(\phi-\phi_{c}\right)^{t}$. The elasticity exponent is expected to be the same as the conductivity exponent ${ }^{15}$ provided only central forces exist between the constituent particle in the network. ${ }^{16}$ By contrast, noncentral forces, or bond bending, break the similarity between conductivity and elasticity, leading to an increase in the elasticity exponent. ${ }^{17}$ For singly connected networks, ${ }^{18}$ the exponent is predicted to be about 3.5 . Experimentally, measurements on beams of sintered metal $^{19}$ yielded $\tau \approx 3.8$ with $t \approx 2.2$, over an equally large range in $\phi-\phi_{c}$. The behavior of the shear rigidity of the network of surfactant micelles is considerably different. This may reflect the different nature of the micelle interactions, which might change $\tau$. Finally, the value of $\phi_{c}$ found here is consistent with that found from simulations of interacting spheres. ${ }^{20}$ It can be expected to change as $E_{a}$ is varied, modifying $\tau_{c}$. These inverted micelles allow the investigation of this important issue.

We conclude by emphasizing that the results presented here demonstrate that the elastic properties of com- plex fluids are both rich and varied, and provide new insights into their dynamics and interactions.

We thank M. Lindsay and J. Sung for assistance and S. Safran, F. Pincus, R. Klein, S. Milner, and T. Witten for fruitful discussions.

${ }^{1}$ P. Eckwell, L. Mandell, and K. Fontell, J. Colloid Interface Sci. 33, 215 (1970).

${ }^{2}$ Y. C. Jean and H. J. Aacke, J. Am. Chem. Soc. 100, 6320 (1978)

${ }^{3}$ M. Zulauf and H. F. Eicke, J. Phys. Chem. 83, 480 (1979).

${ }^{4}$ T. Assih, F. Larche, and P. Delord, J. Colloid Interface Sci. 89, 35 (1982).

${ }^{5}$ J. S. Huang, S. A. Safran, M. W. Kim, G. S. Grest, M. Kotlarchyk, and N. Quirke, Phys. Rev. Lett. 53, 592 (1984).

${ }^{6}$ J. S. Huang, J. Chem. Phys. 82, 480 (1985).

${ }^{7}$ M. Kotlarchyk, J. S. Huang, and S.-H. Chen, J. Chem. Phys. 89, 4382 (1985).

${ }^{8}$ J. S. Huang, X. L. Wu, and J. Sung, J. Colloid Interface Sci. (to be published).

${ }^{9}$ Ping Sheng, in Homogenization and Effective Moduli of Materials and Media, edited by J. L. Ericksen, D. Kinderlehrer, R. Kohn, and J.-L. Lions (Springer-Verlag, New York, 1986), p. 196.

${ }^{10}$ Z. Hashin, J. Appl. Mech. 29, 143 (1962).

${ }^{11}$ J. G. Berryman, J. Acoust. Soc. Am. 68, 1809 (1980).

${ }^{12} \mathrm{R}$. Zallen, The Physics of Amorphous Solids (Wiley, New York, 1983), p. 49.

${ }^{13}$ L. D. Landau and E. M. Lifshitz, Fluid Mechanics (Pergamon, Oxford, 1980).

${ }^{14} \mathrm{D}$. Stauffer, Introduction to Percolation Theory (Taylor and Francis, London, 1985), p. 52.

${ }^{15}$ P. G. de Gennes, J. Phys. Lett. 37, L1 (1976).

${ }^{16}$ S. Alexander, J. Phys. (Paris) 45, 1939 (1984).

${ }^{17}$ S. Feng, P. Sen, B. I. Halperin, and C. J. Lobb, Phys. Rev. B 30, 5386 (1984).

${ }^{18}$ Y. Kantor and I. Webman, Phys. Rev. Lett. 52, 1891 (1984).

${ }^{19}$ D. Deptuck, J. P. Harrison, and P. Zawadzki, Phys. Rev. Lett. 54, 913 (1985).

${ }^{20}$ S. A. Safran, I. Webman, and G. S. Grest, Phys. Rev. A 32, 506 (1985). 